\title{
Laser Cleaning of Silicon Surfaces
}

\author{
Paul Leiderer, Johannes Boneberg, \\ Mario Mosbacher, Andreas Schilling and \\ Oguz Yavas
}

\author{
Fakultät für Physik, Universität Konstanz, D-78434 Konstanz, Germany
}

\begin{abstract}
The continuing trend towards miniaturization of integrated circuits requires increasing efforts and new concepts to clean wafer surfaces from dust particles. We report here about our studies of the "steam laser cleaning" process first described by Tam and coworkers: In order to remove submicron particles from a surface, first a thin liquid layer is condensed onto the substrate from the gas phase, and is subsequently evaporated momentarily by irradiating the surface with a short laser pulse. We have investigated the nucleation and growth of gas bubbles in the liquid, by which the whole process is started, with optical techniques like light scattering and surface plasmon resonance spectroscopy. The experiments indicate that the temperature where nucleation sets in is surprisingly low, which facilitates the application of this phenomenon for cleaning purposes. On the basis of these results and in order to study the cleaning effect for the particularly interesting surface of silicon in a quantitative way, we have deposited well-characterized spherical polymer and silica particles of different diameters from several ten to hundred nanometers on commercial Si wafers and have studied systematically the cleaning efficiency of the explosive evaporation process. The results show that steam laser cleaning is a promising and suitable method for removing sub-micron particles from semiconductor surfaces.
\end{abstract}

Keywords: laser cleaning, bubble nucleation, superheating, pressure measurement, particle removal, ablation

\section{INTRODUCTION}

As the structures on microelectronic chips are getting smaller and smaller, reaching the regime of a hundred nanometers, the removal of submicroscopic particle contaminants on wafer surfaces becomes of increasing importance. Due to the strong influence of adhesion forces acting on small particles the conventional cleaning methods, such as ultrasonics, are inefficient for the removal of submicroscopic contaminants, which gives rise to significant yield losses in the manufacturing process. ${ }^{1,2}$ Recently it has been demonstrated that particles down to diameters of $0.2 \mu \mathrm{m}$ can efficiently be removed by steam laser cleaning. ${ }^{3-5}$ In this laser-cleaning process that motivated the present investigation a thin liquid film is adsorbed onto the contaminated surface, which is then rapidly heated by pulsed laser irradiation. The subsequent explosive evaporation of the liquid film causes the removal of the contaminating particles. The practical relevance of explosive liquid evaporation in steam laser cleaning and other technologically important areas, such as laser surgery, laser chemistry, and localized depassivation of metallic surfaces, ${ }^{6-10}$ has motivated research in the bubble nucleation and pressure generation processes induced by pulsed laser heating of a liquid-solid interface. Initial studies had been performed on thin liquid films using optical transmission probe, ${ }^{11}$ providing useful information on temperature transients and "explosion" thresholds. However, liquid film rupture and droplet formation in the probe beam path aggravate the data analysis and, in addition, the optical transmission probe is limited to certain semitransparent solid substrates. Therefore, in our previous studies we have applied optical reflectance, light scattering, and piezoelectric transducer probes to bulk liquids in contact with solid surfaces. ${ }^{12-15}$ Using these test methods we have been able to clarify several aspects of bubble nucleation at a liquid-solid interface induced by pulsed laser heating. We have for example demonstrated that the optical properties of a foamy liquid layer could be described by an effective-medium theory by Maxwell Garnett ${ }^{16}$ when bubbles are small compared to the probe beam wavelength, and that Mie scattering dominates when bubbles reach a size comparable to the probe beam wavelength, 
resulting in a transient decrease in the specular reflectance signal. Further, threshold fluences for bubble growth to an observable size. i. e., to the order of the probe beam wavelength, could be determined accurately using both optical reflectance and piezoelectric transducer measurements. In addition, from optical reflectance measurements the bubble growth velocities for various liquids on a $0.2 \mu \mathrm{m}$ thick chromium film could be estimated to be of the order of $\mathrm{m} / \mathrm{s}(\approx 2 \mathrm{~m} / \mathrm{s}$ for alcohols, and $=4 \mathrm{~m} / \mathrm{s}$ for water). Bubble growth velocities of the same order were obtained recently by interferometric measurements ${ }^{17}$. Simultaneous measurements of optical temperature probing and specular reflectance have demonstrated that bubble nucleation takes place at moderate liquid superheating ${ }^{18}$, indicating the heterogeneous nature of the nucleation process, where the solid surface provides nucleation sites for bubbles.

Due to the limitations of the previously used test methods, despite their success in the study of the nucleation processes at a liquid-solid interface as briefly described above, some problems still remain unsolved: for example, because of lack of bandwidth and transducer ringing, the piezoelectric transducer technique does not allow an absolute measurement of pressure amplitudes on a nanosecond time scale, providing only qualitative results or requiring tedious calibration procedure. Furthermore, the optical reflectance and light scattering signais are very small as long as the bubbles have not reached a certain size comparable to the probe beam wavelength, and are therefore relatively insensitive for smaller or embryonic bubbles. Here, we will present in chapter 2 the results of our recent studies using surface plasmons as a probe, which proved to be a highly sensitive tool for the study of nucleation and pressure generation processes at a liquid-solid interface induced by pulsed laser heating, providing information also at the early stages of the nucleation process and enabling the measurement of absolute pressure amplitudes on a nanosecond time scale.

In chapter 3 we will describe the state of our investigations on particle removal from silicon surfaces. By using well-defined submicroscopic spheres of different materials one obtains sharp threshold values for the ablation process and can moreover study the effect of successive cleaning pulses in a quantitative way.

\section{LASER-INDUCED VAPOR BUBBLES AND PRESSURE WAVES}

The experimental setup for the surface plasmon probe (SPP) of the laser-induced bubble nucleation at a liquid-solid interface is depicted in Fig. 1(a). A thin silver film of $53 \mathrm{~nm}$ is evaporated in high vacuum onto the base of a heavy flint glass prism ( $\mathrm{n}=1.75$ for $\lambda=632.8 \mathrm{~nm}$ ). A $2 \mathrm{~nm}$ thick chromium interlayer is used to increase the adhesion between the silver film and the glass prism. The prism with the silver film is mounted on a cuvette filled with distilled water. Surface plasmons are excited optically at the silver-water interface via attenuated total reflection (ATR) in the Kretschmann configuration using a $5 \mathrm{~mW}$ HeNe probe laser. ${ }^{19}$ Bubble nucleation takes place at the silver-water interface upon heating by a $\mathrm{KrF}$ excimer laser pulse $\left(\lambda=248 \mathrm{~nm}, \mathrm{FWHM}=25 \mathrm{~ns}\right.$, spot size $\left.1 \times 1 \mathrm{~cm}^{2}\right)$ through a quartz window. Any transient change of the surface plasmon resonance angle due to a change of the dielectric function of the water layer on the silver film induced by bubble nucleation, temperature rise, or pressure changes, is monitored by a fast photodiode (rise time $\leq 1 \mathrm{~ns}$ ). The signal is amplified using an ac-coupled $1 \mathrm{GHz}$ bandwidth amplifier, and recorded on a $500 \mathrm{MHz}$ digitizing storage oscilloscope. As indicated in Fig. 1(b), it is calculated that the appearance of a vapor layer of $1 \mathrm{~nm}$ thickness will cause a significant shift of the surface plasmon resonance to a smaller angle of incidence and thus lead to a transient change in the measured intensity, if the angle of incidence of the probe laser is kept fixed. In order to directly compare the results of the novel SPP with the previously used optical reflectance probe (ORP), a second HeNe probe laser, incident from the front side through the liquid and probing the same spot as the SPP, is used to monitor simultaneously the transient reflectance changes caused by light scattering on bubbles. If not stated otherwise, the SPP measurements presented in the following have been performed by setting the angle of incidence for the probe beam to the middle of the left wing of the surface plasmon resonance, which is located at about $53^{\circ}$ as shown in Fig. 1(b). In order to verify that no reversible changes of the optical constants of the silver film take place upon laser heating, the plasmon resonance curve has been measured prior to and after each series of experiments.

As already mentioned, several effects may influence the position and shape of the surface plasmon resonance ${ }^{20}$. In order to determine the contribution of the temperature rise in the silver film, experiments have been conducted first with the bare silver film in the absence of water. Only a slight transient decrease in the reflected intensity is observed in this case when the laser pulse heats the film, due to the combined effect of plasmon resonance shift and broadening. When the experiment is carried out with the cuvette filled with water, on the other hand, the amplitude of the reflectance drop is drastically increased. As calculations show the change of the optical properties of the dielectric half space (in our case water) due to a given temperature rise ${ }^{21}$ causes a much larger shift of the surface plasmon resonance than a change of the optical properties 

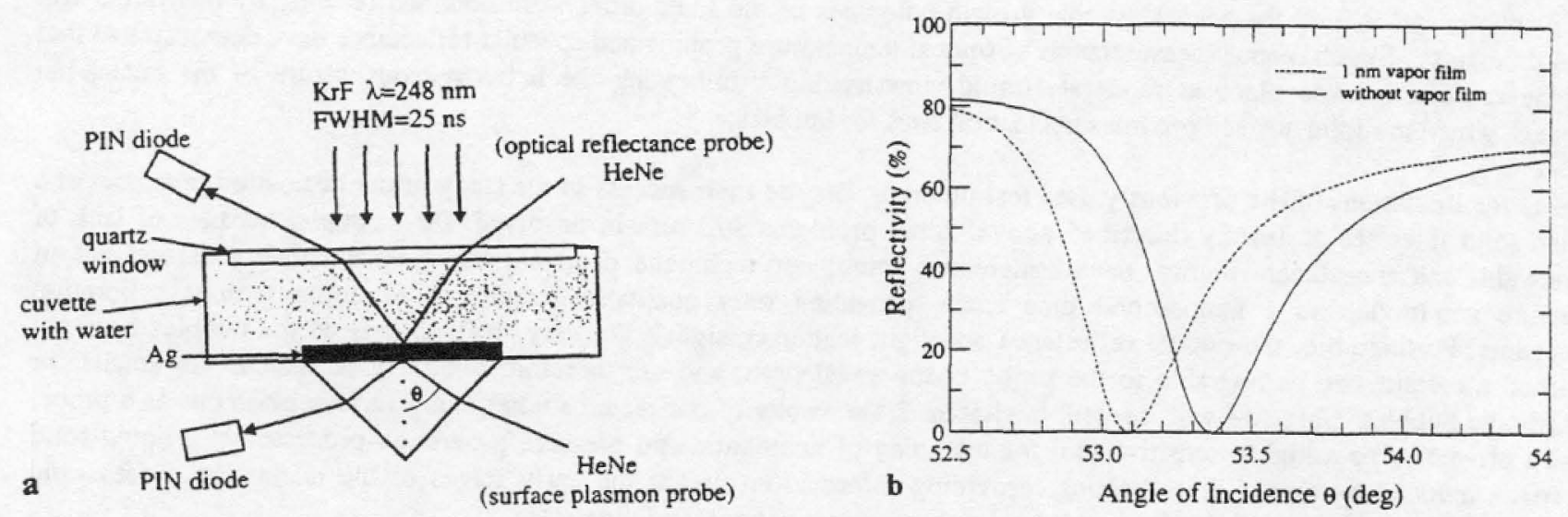

Fig. 1: (a) Experimental configuration for the nanosecond time-resolved study of pulsed laser-induced bubble formation at a liquid-solid interface using the surface plasmon probe (SPP) and optical reflectance probe (ORP) technique. (b) Calculated shift of the surface plasmon resonance curve caused by a $1 \mathrm{~nm}$ thick vapor film at the water-silver interface.

of the silver film ${ }^{22}$ induced by the same temperature rise. Consequently, the resulting shape of the SPP is predominantly governed by the changes of the refractive index of water with temperature.

\subsection{Nucleation threshold}

As long as the laser fluence is below a certain value (which corresponds to the threshold for bubble nucleation, see below), the SPP signal exhibits no particular extra features, as shown in the topmost trace of Fig. 2(a). When the excimer laser fluence is increased further, however, an additional structure in the SPP signal is observed as seen in the lower traces of Fig. 2(a). A hump in the reflectance drop starts to appear at an excimer laser fluence of $F=10.5 \mathrm{~mJ} / \mathrm{cm}^{2}$, and becomes more pronounced with increasing laser fluence. The appearance of this hump can be interpreted as the onset of bubble nucleation at the water-silver interface. The surface plasmons are effectively scattered by the bubbles, and consequently the surface plasmon resonance is broadened and shifted, resulting in a temporary increase of the SPP intensity. When the bubbles collapse, the surface plasmon resonance becomes again narrower and the SPP signal decreases to a level which is given by the resonance shift due to the actual temperature present at the water-silver interface. For the laser fluence of $F=10.5$ $\mathrm{mJ} / \mathrm{cm}^{2}$ numerical computations yield a peak temperature of $T=384 \mathrm{~K}$ at the water-silver interface, i.e., a surprisingly low liquid superheating of about $10 \mathrm{~K}$. This superheating is of the same order as recently concluded for a similar system. ${ }^{18}$ While the hump in the SPP signal starts to appear at $F=10.5 \mathrm{~mJ} / \mathrm{cm}^{2}$ and becomes more pronounced with increasing laser fluence the simultaneously acquired ORP signals exhibit no particular features up to a fluence level of $F=19.5 \mathrm{~mJ} / \mathrm{cm}^{2}$. Above thi: threshold fluence, for which computations predict a peak temperature of $T=473 \mathrm{~K}$ at the water-silver interface (liquic superheating $\approx 100 \mathrm{~K}$ ), a transient decrease in the ORP signal starts to appear as shown in Fig. 2(b). This result strikingl: demonstrates the high sensitivity of the surface plasmon probe to small bubbles which collapse before they grow up to the order of the probe beam wavelength and therefore remain invisible to the ORP. The difference in the threshold fluence: acquired using these two different methods can be explained by assuming that the threshold temperature obtained using the SPP corresponds to the temperature where bubble nucleation sets in, and the threshold temperature obtained using the ORI corresponds to the temperature where bubble growth becomes effective. 


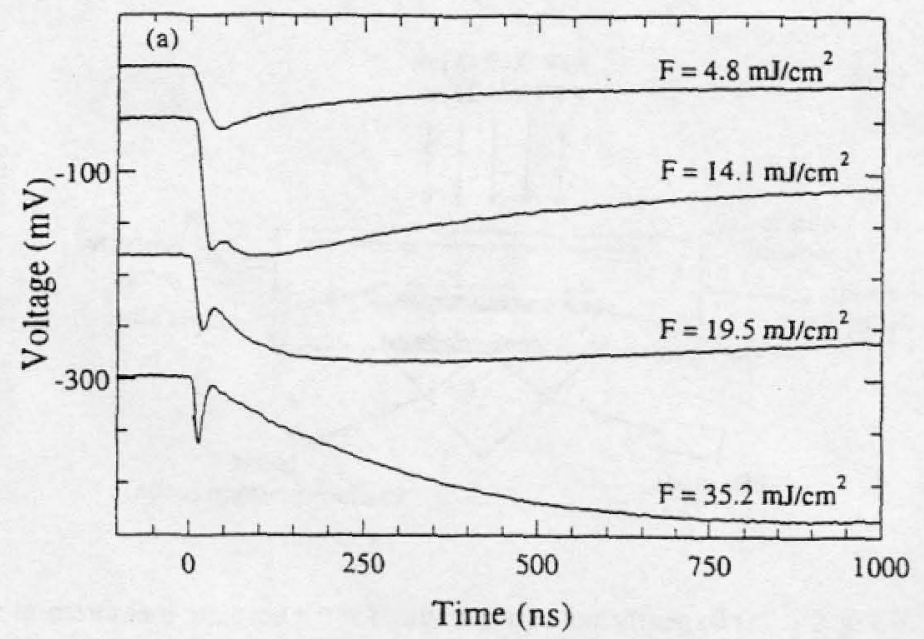

Fig. 2: (a) Surface plasmon probe and (b) optical reflectance signals for a water-silver interface with increasing excimer laser fluence. $10 \mathrm{mV}$ corresponds to a reflectance change of $3 \%$. The curves are offset for clarity.

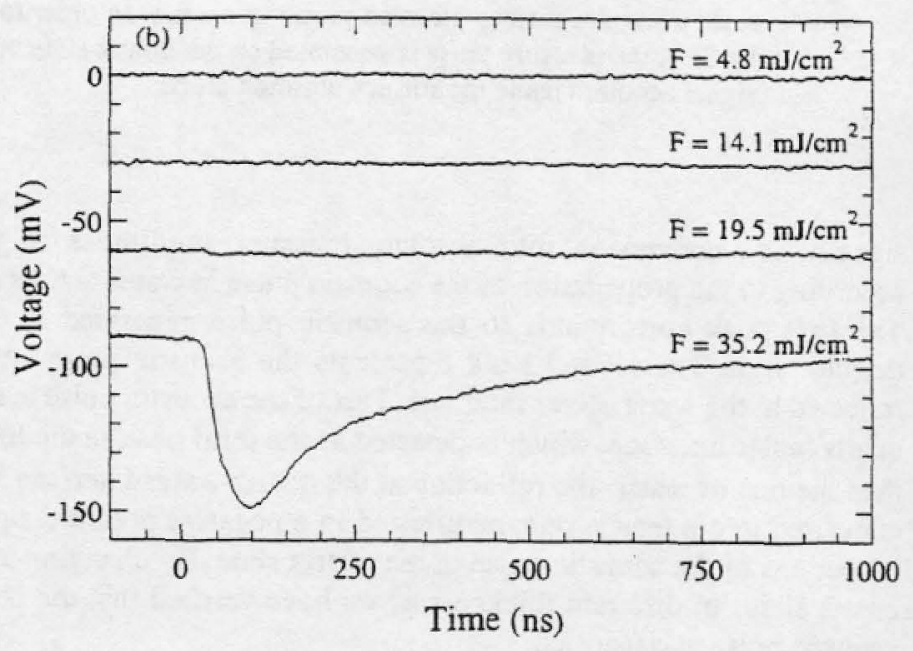

\subsection{Bubble induced pressure waves}

In our previous studies using piezoelectric transducers we have demonstrated the generation of high intensity acoustic pulses on a nanosecond time scale due to rapid bubble growth. ${ }^{13-15}$ This enhancement of the acoustic pulse intensity by the rapid bubble growth process is thought to play a major role in the removal of submicroscopic particles during laser cleaning. Since the piezoelectric transducer measurements could not provide the absolute pressure amplitudes, a quantitative data analysis was not possible yet.

Recently, however, we have succeeded to apply the surface plasmon probe also for absolute pressure measurements on a nanosecond time scale. ${ }^{23.24}$ In order to eliminate any temperature effects, the experimental setup presented in Fig. 1 has been slightly modified as shown in Fig. 3(a). A thin quartz slide is placed in water at variable distance from the prism. The quartz slide is coated with $20 \mathrm{~nm}$ chromium and $60 \mathrm{~nm}$ silver on its surface facing the prism. The irradiation of the quartz slide from the rear side results in a sudden heating of the silver film and in bubble nucleation at the silver-water interface. The acoustic pulse generated during this process propagates toward the prism and can be detected by the SPP. The known relation for the dielectric constant for water as a function of pressure ${ }^{21}$ is utilized to extract the absolute pressure amplitudes from the shift of the surface plasmon resonance curve. An example for the transient pressure signal acquired using this setup is presented in Fig. 3(b). A detailed description of the data acquisition system and the analysis of the SPP 

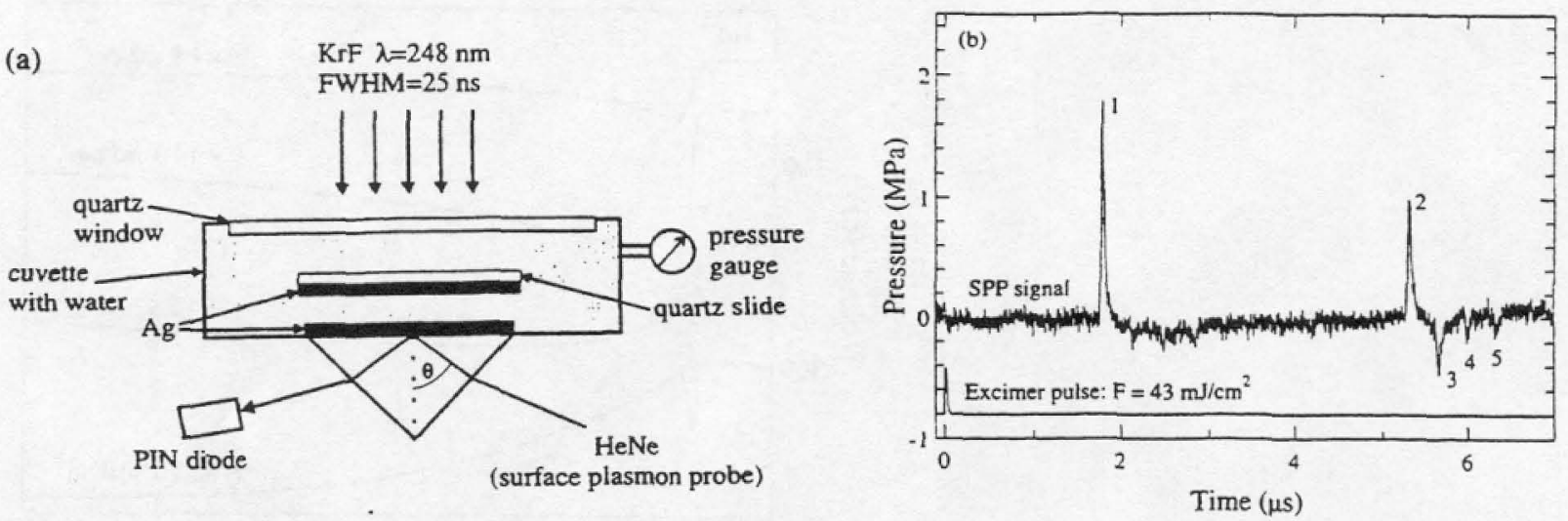

Fig. 3: (a) Experimental configuration for the absolute measurement of acoustic pulse amplitudes on a nanosecond time scale using the surface plasmon probe technique. In order to eliminate thermal effects on the silver film probed by SPP, the pressure pulse is generated on the quartz slide upon excimer laser irradiation. (b) Transient pressure signal acquired using the surface plasmon probe.

signals, i.e., conversion into absolute pressure amplitudes is given elsewhere. ${ }^{23 .} 24$ The signal exhibits several peaks according to the propagation of the acoustic pulse in water and its repeated reflection between the prism and the quartz slide. The first peak corresponds to the acoustic pulse generated at the silver-water interface after its propagation to the SP detector film. The second peak represents the acoustic pulse after its round-trip between the prism and the quartz slide, reflected at the water-silver interface. Part of the acoustic pulse is transmitted into the quartz slide and is reflected back at the quartz-water interface, which is detected as the third peak in the SPP signal. Since the acoustic impedence of quartz is higher than the one of water, the reflection at the quartz-water interface leads to a phase change of $\pi$, and the compressive wave is converted into a tensile one, manifested in a negative pressure signal. The successive peaks 4 and 5 are caused by multiple reflections of the acoustic pulse in the quartz slide. By changing the distance of the quartz slide from the prism, and by using quartz slides of different thicknesses, we have verified that the peaks in the SPP signal represent multiple reflections of the acoustic pulse as described.

The width of the acoustic pulse is measured to be about $40 \mathrm{~ns}$, which is much shorter than the previously measured pressure pulse width of $\approx 100 \mathrm{~ns}$ using photoacoustic probe beam deflection technique ${ }^{25}$ demonstrating the improved time resolution of the SPP. Due to the localized probe depth $(\approx 200 \mathrm{~nm})$, the SPP provides the real pulse profiles, while the photoacoustic deflection probe detects generally a larger pressure pulse width because of its integrating nature, i.e., the finite probe beam waist and the interaction length of the probe beam. According to our measurements the peak amplitude of the initial pulse amounts to $\approx 2 \mathrm{MPa}$ for the laser irradiation using $F=43 \mathrm{~mJ} / \mathrm{cm}^{2}$, and in the successive echoes its intensity gradually decreases due to reflection and absorption losses. A pressure amplitude of the same order, but about two to three times lower, has been previously measured for a similar system using photoacoustic probe beam deflection and piezoelectric transducer techniques. ${ }^{25}$ The small discrepancy in the measured pressure amplitudes can be attributed to the different samples used and to the difference in the temporal resolution of the test methods.

\subsection{Direct proof for laser-induced bubbles}

The fact that the reflection of the acoustic pulse at an acoustically less dense medium leads to a phase change, i.e., to the conversion of the compressive pulse into a tensile one, could be used to directly prove the generation of bubbles by the excimer laser heating of the water-silver interface. As long as a bubble layer is present at the water-silver interface on the 
quartz slide, the acoustic pulse will be reflected as a tensile wave, since the reflection will take place at a water-vapor interface. When bubbles collapse and the reflection takes place at the water-silver interface, the reflected pulse will be compressive. This has been verified by placing the quartz slide at close distance from the prism and successively increasing the distance after each excimer laser pulse. Representative results are depicted in Fig. 4 and clearly demonstrate the existence of bubbles up to several hundred ns following the excimer laser pulse, manifested by a tensile reflected acoustic pulse. Although our previous studies already had delivered the clear evidence for laser-induced bubbles, the results presented here provide a more direct proof for such bubbles at a liquid-solid interface. The amplitude of the reflected pressure pulse as a function of the distance $d$ between the quartz slide and the sample surface (first echo) is plotted in Fig. 5 . At $\mathrm{d}=180 \mu \mathrm{m}$ (the smallest distance that could be achieved experimentally), corresponding to a delay time of about $240 \mathrm{~ns}$, the pressure amplitude has a negative value, as already seen in Fig. 4(a). As the distance is increased, the pressure peak drops in magnitude, changes sign around $d=0.5 \mathrm{~mm}$, and then approaches a positive saturation value. We interpret this dependence as a gradual decrease of the bubble size and/or number, which already proceeds after $240 \mathrm{~ns}$, and a complete collapse of the bubbles when the signal reaches saturation after about $1.8 \mu$ s.

It should be pointed out at this point that the bubble behavior described above is characteristic for bulk liquid in contact with a solid surface. Studies with a thin liquid film have to be carried out yet.

Fig. 4: (a) Direct proof of bubbles by the phase reversal of the acoustic pulse upon reflection at the water-silver interface when bubbles are present. The distance $d$ between the two plates is $180 \mu \mathrm{m}$, and the delay time between the first and the second (in this case inverted) peak is $240 \mathrm{~ns}$, corresponding to one round-trip of the acoustic pulse. (b) On a longer time scale, when bubbles are collapsed, reflection takes place without phase reversal. $d=1.3 \mathrm{~mm}$ in this case.
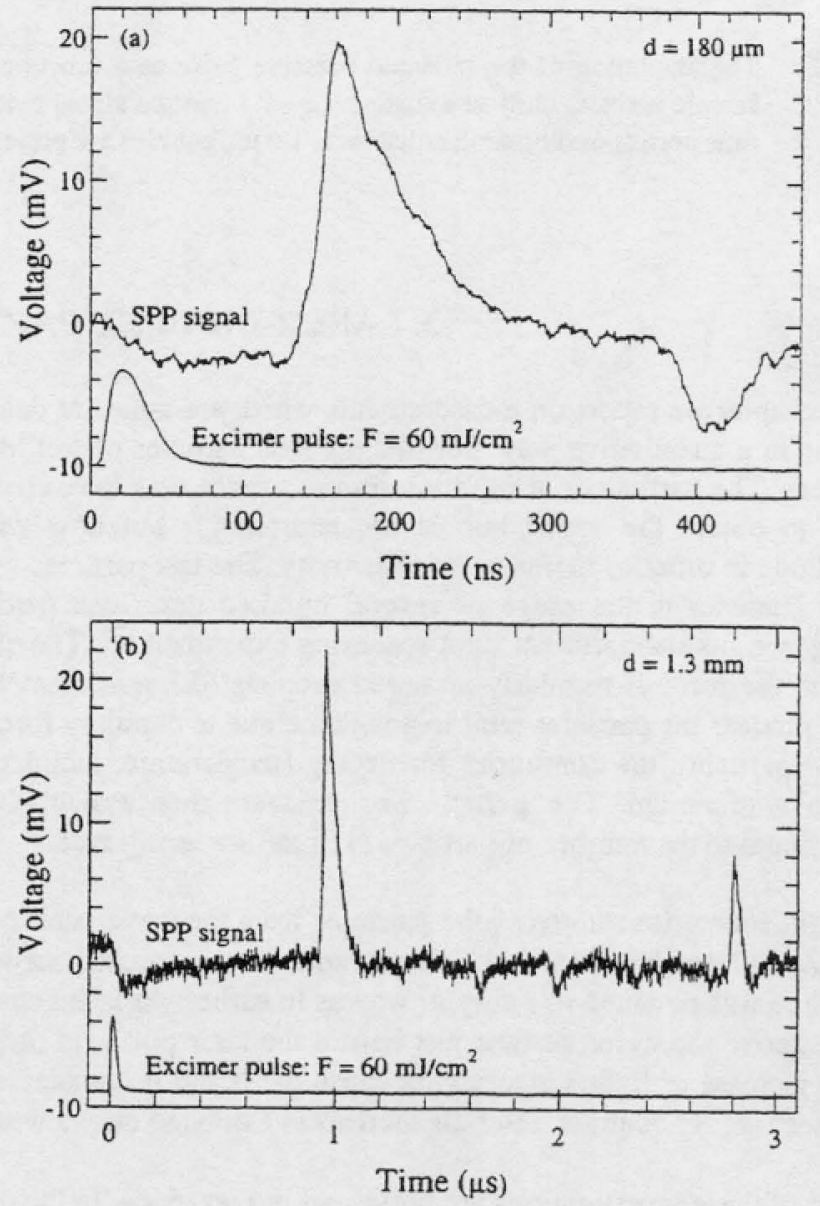


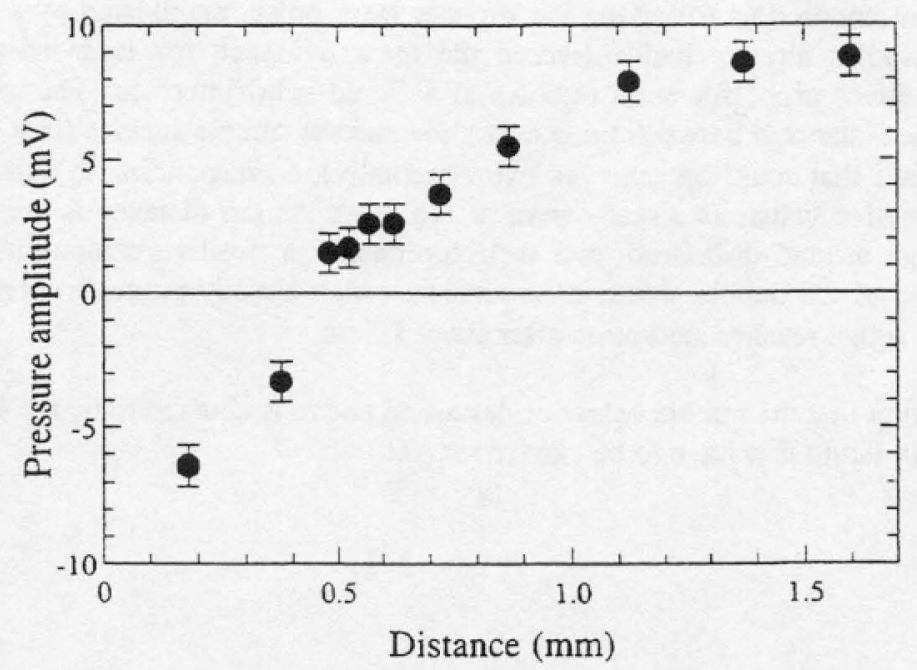

Fig. 5: The amplitude of the reflected pressure pulse as a function of the distance of the quartz slide from the SPP sample surface. Only at a distance $d \sim 1.3 \mathrm{~mm}$ the signal reaches saturation, indicating that up to the round-trip time corresponding to that distance, $1.8 \mu \mathrm{s}$, bubbles are present after their generation by the excimer puise.

\section{LASER-INDUCED PARTICLE REMOVAL}

In this chapter we report on measurements which are aimed at determining the efficiency of particle removal by steam laser cleaning in a quantitative way. For this purpose particles of well-defined size and shape were first deposited on commercial $\mathrm{Si}$ wafers. The particle concentration on the surface was measured via light scattering with a set-up similar to the one used earlier to obtain the vapor bubble concentration ${ }^{12}$, but now with a photomultiplier as detecting element instead of a photodiode in order to increase the sensitivity. The test particles were small monodisperse silica or polystyrene (PS) spheres with a diameter in the range of several hundred $\mathrm{nm}$. Such particles are commercially available in aqueous suspension, serving, e.g., as standards for light scattering experiments ${ }^{26}$. The particular suspension was then deposited on a wafer surface either in the form of regularly arranged droplets $\left(0.2 \mathrm{~mm}\right.$ diameter) or as a large drop covering about $1 \mathrm{~cm}^{2}$. During the drying process the particles tend to coagulate due to capillary forces. Since we are interested here mainly in the behavior of isolated particles, the conditions for drying (temperature, inclination of the wafer) were chosen such that coagulation was kept to a minimum. The particle positions are then essentially uncorrelated, and the scattered intensity I is directly proportional to the number of particles $\mathrm{N}$ in the scattering area.

The light source for removing the particles from the waver surface was in these experiments a frequency-doubled Nd:YAG laser ( $\lambda=532 \mathrm{~nm}, \mathrm{FWHM}=7 \mathrm{~ns}$ ). The irradiated areas varied between 1 and $50 \mathrm{~mm}^{2}$. The liquid film for the steam cleaning procedure was obtained in a similar way as in earlier work ${ }^{3}$ by condensing a burst of water/alcohol vapor supplied through a nozzle above the wafer surface just before the laser pulse. In the areas covered by test particles this condensation led to a strong increase of light scattering, because the liquid accumulating around the spheres gives rise to an enhanced scattering cross section. By contrast, laser-cleaned areas exhibited only a weak scattering signal upon vapor condensation.

Results of these investigations are presented in Figs. 6 - 9. In the case of a surface carrying a regular trace of particle patches (each containing several hundred spheres), the test light from a $5 \mathrm{~mW} \mathrm{He}-\mathrm{Ne}$ laser was focussed to a diameter of 

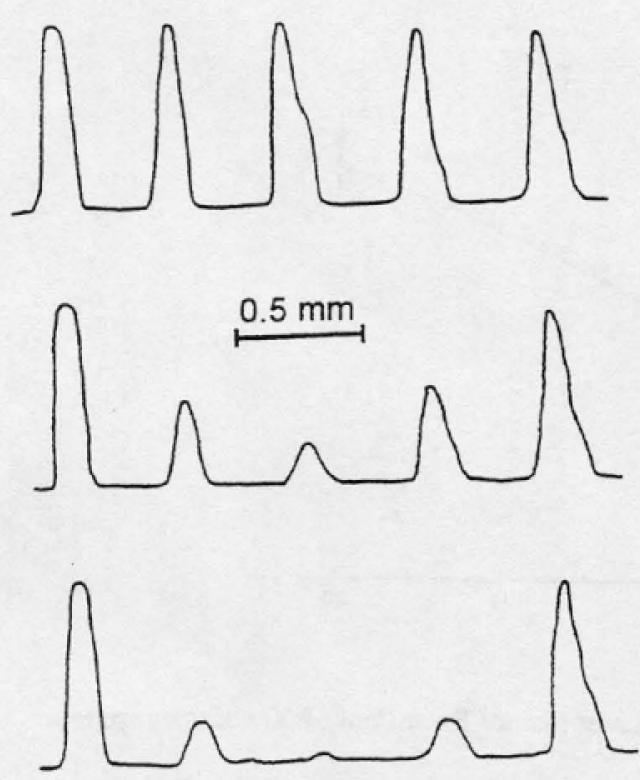

:Fig. 6 Light intensity scattered from a row of particle dots consisting of colloidal particles (800 nm PS spheres) on a silicon wafer. (a) as prepared; (b) after 1 cleaning pulse; (c) after 3 cleaning pulses.

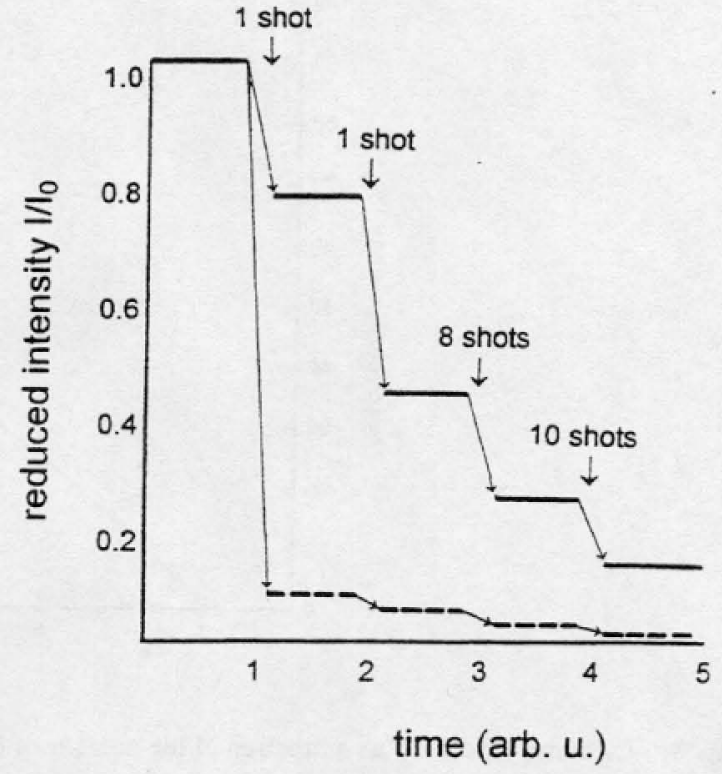

Fig. 7: Removal of $800 \mathrm{~nm}$ silica particles from a Si surface by a sequence of laser pulses. The reduced scattered intensity $\quad I_{0}$ is approximately proportional to the fraction $\mathrm{N}_{\mathrm{r}} / \mathrm{N}_{0}$ of particles remaining on the surface. The fluences of the pulses were 152 (full line) and $180 \mathrm{~mJ} / \mathrm{cm}^{2}$ (dashed line), respectively.

$0.1 \mathrm{~mm}$ and repetitively scanned along a row of particle dots, yielding signals as displayed in Fig. 6. Fig. 6a shows a signal of the "as prepared" sample; Figs. $6 \mathrm{~b}$ and $6 \mathrm{c}$ represent the same row after it had been exposed to 1 and 3 cleaning pulses, respectively. The effect of particle removal is clearly visible.

For the more systematic measurements we used somewhat smaller particle concentrations, distributed homogeneously across the wafer surface. The He-Ne test laser beam was now kept at a fixed position, illuminating a spot with a diameter of $0.5 \mathrm{~mm}$ and a total number of again on the order of some hundred particles. The beam of the cleaning laser had a diameter of $5 \mathrm{~mm}$, i. e. much larger than the test beam, so that the laser fluence in the monitored area can be considered as homogeneous.

Fig. 7 shows the reduction in the scattered intensity (and hence in the particle number) for a sequence of laser pulses at two different fluence levels. Silica spheres with a diameter of $800 \mathrm{~nm}$ were used as test particles in this case. At a laser fluence of $152 \mathrm{~mJ} / \mathrm{cm}^{2}$ the first pulse removes 25 percent of the particles, whereas at the somewhat increased level of $180 \mathrm{~mJ} / \mathrm{cm}^{2}$ already more than 90 percent are detached under otherwise identical conditions.

If the majority of the particles is bound to the surface with the same energy, as one might expect it for monodisperse spheres, the probability $\mathrm{P}$ that a particle is removed by an individual laser pulse should only depend on the laser fluence $F$ and not on the previous pulses. Hence when a sequence of $n$ pulses is applied, the fraction of remaining particles $N_{r} / N_{0}$ (where $N_{0}$ is the initial particle number) should be given by

$$
\mathrm{N}_{\mathrm{r}} / \mathrm{N}_{\mathrm{O}}=(1-\mathrm{P})^{\mathrm{n}}
$$




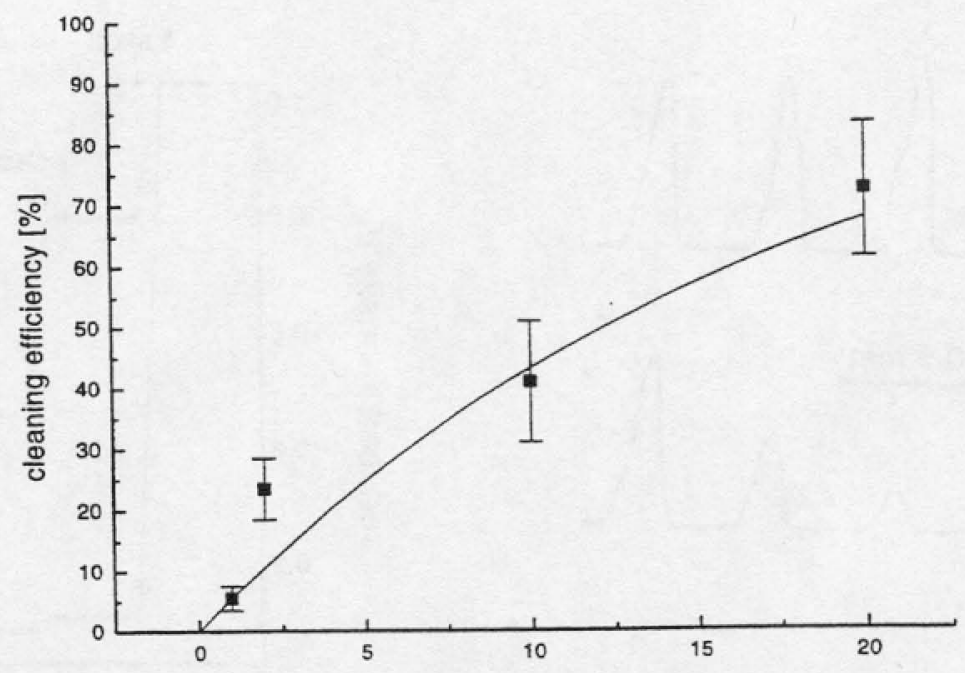

Fig. 8: Cleaning efficiency as a function of the number of laser pulses. Laser fluence $\mathrm{F}=\mathrm{mJ} / \mathrm{cm}^{2} ; 800 \mathrm{~nm}$ silica spheres.

This is indeed confirmed experimentally as demonstrated in Fig. 8, where we have plotted data for $\left(1-\mathrm{I}_{\mathrm{o}}\right)$ vs. the number of applied laser pulses, for a laser fluence of $115 \mathrm{~mJ} / \mathrm{cm}^{2}$ per pulse. ( $\mathrm{I}_{0}$ is here the initial scattering intensity.) According to the argument given earlier the quantity $\mathrm{I}_{0}$ is equivalent to the fraction of remaining particles. Hence $\left(1-I / I_{0}\right)$ is the fraction of removed particles, which we denote in the following as "cleaning efficiency". Using Eq. (1) one expects that $\left(1-\mathrm{I} / \mathrm{I}_{0}\right)$ should vary as $\left(1-(1-P)^{n}\right)$. The full line in Fig. 8 represents the quantity $\left(1-(1-P)^{\mathrm{n}}\right)$ with $\mathrm{P}$ taken here to be 0.05 , as derived from the datum point for the first pulse. The agreement between the data and this line is quite satisfactory. Therefore the ablation process for our samples appears to be well-controlled, making quantitative studies feasible.

In Fig. 9 we show results for the energy dependence of particle removal by the steam cleaning process. Plotted is the percentage of ablated particles for $1,2,10$ and 20 laser pulses, respectively. A sharp threshold at $F=110 \mathrm{~mJ} / \mathrm{cm}^{2}$ is

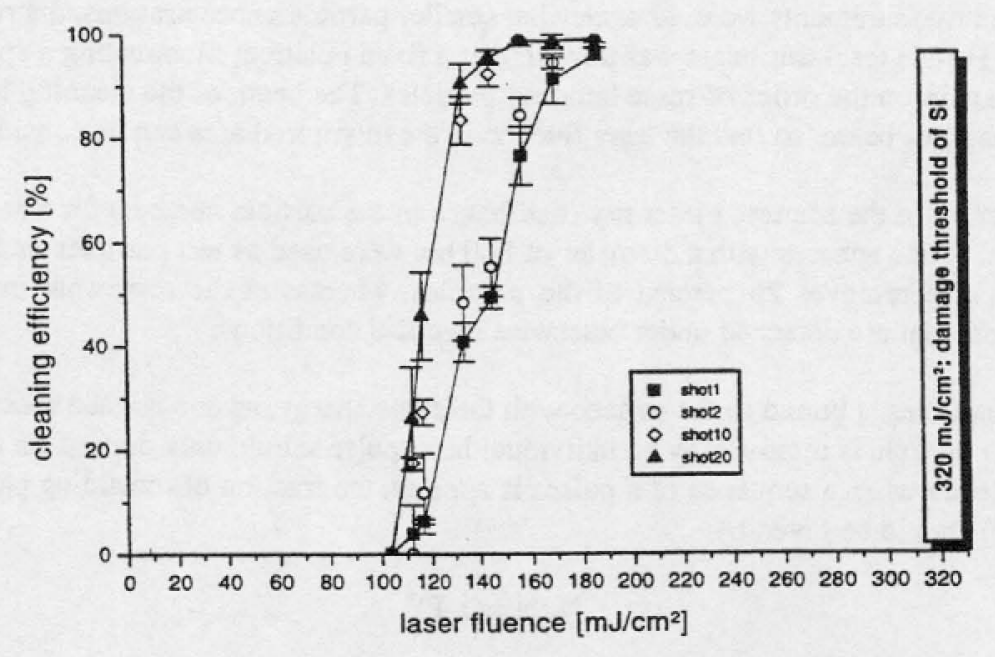

Fig. 9: Cleaning efficiency for $800 \mathrm{~nm}$ silica spheres as a function of laser fluence for $1,2,10$ and 20 laser pulses. 
observed, below which no particle removal takes place. On the other hand, for fluences above $170 \mathrm{~mJ} / \mathrm{cm}^{2}$ more than 90 percent of the particles are removed by the first laser pulse. If more pulses are applied, the integrated cleaning efficiency is increased, as already suggested by Fig. 7, and reaches nearly 100 percent at the higher energy values. For comparison, the threshold value where a (bare) Si surface starts to melt is $320 \mathrm{~mJ} / \mathrm{cm}^{2}$.

Results similar to the one in Fig. 9 were obtained also for the $800 \mathrm{~nm}$ PS particles. In particular, the threshold value for the ablation was the same as for the silica spheres. The slope of the ablation curve just above threshold, however, was somewhat larger in the case of PS, indicating a smaller binding energy than for the silica particles. From the identical threshold values $F_{\text {th }}$ we conclude that the threshold fluence is determined by the onset of explosive evaporation of the adsorbed film, which is of course the same in both cases. Preliminary experiments were also carried out with other particle sizes down to as small as $\mathrm{d}=60 \mathrm{~nm}$, leading again to the same threshold value as in Fig. 9.

Finally, we would like to mention an observation concerning the ablation behavior of particle aggregates, as they develop partly during the preparation process as dimers, trimers and also larger clusters. By optical microscopy between subsequent laser pulses we found that such aggregates are typically not removed as a whole in one shot. Rather they are broken up step by step, with individual particles being detached, for which the ablation probability appears to be higher than for the complete aggregate.

\section{CONCLUSIONS}

The experiments carried out here have confirmed that steam laser cleaning is an efficient method for removing submicron particles from wafer surfaces. The process of vapor bubble nucleation at the liquid-solid interface, which leads to sufficiently high pressures below the contaminant particle to detach it from the surface, was studied in its initial stages with the sensitive technique of surface plasmon resonance spectroscopy. The results show that already modest superheating gives rise to bubble nucleation, so that the heating of the surface can remain well below the damage threshold. In order to study the particle ablation process we have used well-defined test particles and were able to show that quantitative experiments with small data scatter are possible. Yet these are only the first measurements, and apparently further investigations are required to evaluate the limits of this cleaning strategy and to optimize the parameters such as the laser wavelength and the composition of the adsorbed liquid film. Already now it is obvious, however, that this method is well-suited for preparing extremely clean surfaces as they are necessary for the further miniaturization of integrated circuits.

\section{ACKNOWLEDGEMENTS}

We gratefully acknowledge contributions by many colleagues to the investigations reported here, in particular by J. Bischof, C.P. Grigoropoulos, S. Herminghaus, H.K. Park, and A. Tam. We also thank Wacker Siltronic AG for supplying the silicon substrates used here. Part of this work was supported by the Optik-Zentrum Konstanz.

\section{REFERENCES}

1. S.A. Hoenig, in Particles on Surfaces, ed. K.L. Mittal (Plenum, New York, 1988), Vol. 1, p. 3.

2. T. Hattori, Sol. State Tech., 8 (July, 1990).

3. W. Zapka, W. Ziemlich, and A.C. Tam, Appl. Phys. Lett. 58, 2217 (1991).

4. A.C. Tam, W.P. Leung, W. Zapka, and W. Ziemlich, J. Appl. Phys. 71, 3515 (1992).

5. H.K. Park, C.P. Grigoropoulos, W.P. Leung, and A.C. Tam, IEEE Trans. Comp., Pack., and Manuf. Techn. A 17, 631 (1994).

6. J.A. Parrisch and T.F. Deutsch, IEEE J. Quant. Electr. 20, 1386 (1984).

7. D. Bäuerle, Chemical Processing with Lasers (Springer,Berlin, 1996).

8. O. Yavas, R. Oltra, and O. Kerrec, Appl. Phys. A 63, 321 (1996)

9. R. Oltra, G.M. Indrianjafy, and J.P. Boquillon, J. de Phys.IV C7, 769 (1991).

10. R. Oltra and O. Yavas, Appl. Surf. Sci. 108, 219 (1997) 
11. P.T. Leung, N. Do, L. Klees, W.P. Leung, F. Tong, L. Lam, W. Zapka, and A.C. Tam, J. Appl. Phys. 72, 2256 (1992).

12. O. Yavas, P. Leiderer, H.K. Park, C.P. Grigoropoulos, C.C. Poon, and A.C Tam, Phys. Rev. Lett. 70, 1830 (1993).

13. O. Yavas, P. Leiderer, H.K. Park, C.P. Grigoropoulos, C.C. Poon, and A.C Tam, Appl. Phys. A 58, 407 (1994).

14. O. Yavas, P. Leiderer, H.K. Park, C.P. Grigoropoulos, C.C. Poon, and A.C Tam, Phys. Rev. Lett. 72, 2021 (1994).

15. O. Yavas, Laserinduzierte Gasblasennukleation (Hartung-Gorre, Konstanz, 1994).

16. J.C. Maxwell Garnett, Phil. Trans. Roy. Soc. London 203, 385 (1904).

17. D. Kim, H.K. Park, and C. P. Grigoropoulos, Interferometric study on the growth of pulsed-laser-generated submicron bubble layer on a solid surface, National Heat Trans. Conf. Proc. Vol. 4, pp. 69-77, Houston, Texas (1996)

18. H.K. Park, C.P. Grigoropoulos, C.C. Poon, and A.C Tam, Appl. Phys. Lett. 68, 596 (1996).

19. E. Kretschmann and H. Raether, Z. Naturf. A 23, 2135 (1968).

20. S. Herminghaus and P. Leiderer, Appl. Phys. A 51, 350 (1990).

21. P. Schiebener, J. Straub, J.M.H. Levelt Sengers, and J.S. Gallagher, J. Phys. Chem. Ref. Data 19, 677 (1990).

22. P.B. Johnson and R.W. Christy, Phys. Rev. B 6, 4370 (1972).

23. A. Schilling, O. Yavas, J. Bischof, J. Boneberg, and P. Leiderer, Appl. Phys. Lett.69, 4159 (1996)

24. O. Yavas, A. Schilling,J. Bischof, J. Boneberg, and P. Leiderer, Laser Phys. 7, 343 (1997)

25. H.K. Park, D. Kim, C.P. Grigoropoulos, and A.C. Tam, J. Appl. Phys. 80, 1 (1996)

26. Bangs Laboratories, Inc., 9025 Technology Drive, Fishers, IN 46038-2886, USA. 